\title{
Impact of Small Phonon Energies on the Charge-Carrier Lifetimes in Metal-Halide Perovskites
}

\author{
Thomas Kirchartz ${ }^{1,2, *}$, Tom Markvart ${ }^{3,4}$, Uwe Rau ${ }^{1}$, David A. Egger ${ }^{5, *}$ \\ ${ }^{1}$ IEK5-Photovoltaics, Forschungszentrum Jülich, 52425 Jülich, Germany \\ ${ }^{2}$ Faculty of Engineering and CENIDE, University of Duisburg-Essen, Carl-Benz-Str. 199, 47057 Duisburg, \\ Germany \\ ${ }^{3}$ Centre for Advanced Photovoltaics, Czech Technical University, 16636 Prague 6, Czech Republic \\ ${ }^{4}$ Engineering Sciences, University of Southampton, Southampton SO17 1BJ, UK \\ ${ }^{5}$ Institute of Theoretical Physics, University of Regensburg, 93040 Regensburg, Germany
}

\begin{abstract}
Metal-halide perovskite (MHP) solar cells exhibit long non-radiative lifetimes as a crucial feature enabling high efficiencies. Long non-radiative lifetimes occur if the transfer of electronic into vibrational energy is slow due to, e.g., a low trap density, weak electronphonon coupling or the requirement to release a many phonons in the electronic transition. Here, we combine known material properties of MHPs with basic models for electron-phonon coupling and multiphonon-transition rates in polar semiconductors. We find that the low phonon energies of $\mathrm{MAPbI}_{3}$ lead to a strong dependence of recombination rates on trap position, which we deduce from the underlying physical effects determining non-radiative transitions. This is important for non-radiative recombination in MHPs, as it implies that they are rather insensitive to defects that are not at midgap energy, which can lead to long lifetimes. Therefore, the low phonon energies of MHPs are likely an important factor for their optoelectronic performance.
\end{abstract}


TOC Figure

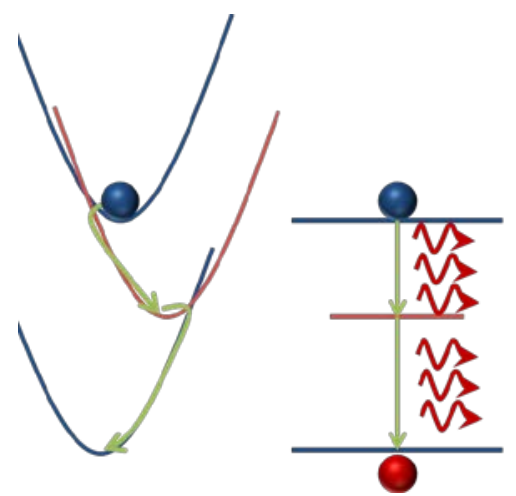


The high open-circuit voltages ${ }^{1-5}$ and long non-radiative charge-carrier lifetimes ${ }^{6-11}$ are key to understanding the exceptional ${ }^{3,12-15}$ photovoltaic performance that has been reached using metal-halide perovskite (MHP) absorber materials. Understanding the reasons for these long lifetimes is of crucial importance because it would help develop the perovskite-based solar cell technology further by directing the discovery of alternative MHP compounds. These may have higher stability 1,3,16,17 than the original methyl-ammonium lead halide perovskites, while retaining the properties that enable long charge-carrier lifetimes. In addition, a clear identification of what microscopic properties of a material are beneficial for long lifetimes would also enable us to screen materials computationally for these desired properties. ${ }^{18-20}$

Among the early proposed explanations for the long lifetimes in MHPs was the observation that in density functional theory calculations most intrinsic defects (vacancy, interstitial, and substitutional defects) are either rather shallow, or have a high formation energy, and are thus unlikely to form. ${ }^{18,21}$ Since then, there have been various additional suggestions attempting to explain how peculiar properties of lead-halide perovskites could lead to the long lifetimes. Among these suggestions is the existence of an indirect band gap ${ }^{22-}$ ${ }^{25}$ just below the direct one, the low density of states at the valence-band maximum, ${ }^{26}$ and the formation of large polarons ${ }^{27,28}$ that were thought to be screened from each other, thereby slowing down recombination. Rather than adding a new explanation, we here intend to estimate the charge-carrier lifetimes (for a given trap density) of MHPs based on existing theories $^{29-33}$ for non-radiative recombination via the emission of multiple phonons.

In general, we consider that recombination of electron-hole pairs in semiconductors may proceed either in a radiative process via the emission of photons, or in a non-radiative process via the emission of phonons or by the Auger effect which also leads to phonon emission eventually. Radiative and Auger-driven recombination are intrinsic mechanisms that occur also in a perfect, defect free single crystal. ${ }^{34,35}$ Band-to-band recombination of an electronhole pair via the emission of multiple phonons, however, is typically considered impossible in 
inorganic semiconductors. Thus, in most thin films of polycrystalline semiconductors the interruption of the crystal periodicity at grain boundaries, or the presence of intrinsic/extrinsic point defects, leads to the creation of localized states in the band gap. The existence of these states is typically thought to accelerate recombination by reducing the number of phonons that have to be emitted in a single transition. Similarly, a commonly stated but infrequently explained rule of semiconductor physics is that this acceleration of recombination events is strongest if the energy of localized states is situated around midgap. Recombination via such (singly charged) localized states in the band gap is typically described by Shockley-Read-Hall $(\mathrm{SRH})^{36,37}$ statistics. While the SRH model explains why recombination at a certain voltage is most likely proceeding via localized states that are between the two quasi-Fermi levels, ${ }^{38-40}$ it does not explain why recombination via midgap energy states would be any different from recombination via other defect levels in between the two quasi-Fermi levels. ${ }^{38}$ To better understand these phenomena and reveal their effect in MHP solar cells, we study the effect of electron-phonon coupling and the phonon energy, i.e., the number of phonons required for a certain transition, on SRH lifetimes via multiphonon emission.

Coordination coordinate diagrams, as shown in Fig. 1, serve as the conceptual starting point for theories of multiphonon transitions in semiconductors or molecules. Let us assume, for instance, a transition of an electron in the conduction band to an available localized state in the band gap. These two states in general correspond to two different potential energy surfaces represented by the upper and lower parabola reflecting different configurations of the crystal (or the molecule) with minima shifted relative to each other on the coordination coordinate axis. The two states relevant for the transition we are considering are vibrational eigenstates of the lower and upper parabola. Recombination may now occur if the overlap between the lowest-energy wavefunction on the upper parabola overlaps with that of the highest-energy wavefunction on the lower one. Depending on the temperature and relative energetics, different paths from upper to lower parabola can be conceived, including 
thermally activated processes. Typically, the relative shift of the two parabolas relative to each other is described by the parameter $S_{\mathrm{HR}}$, the Huang-Rhys factor. It is defined using the energy difference between the minimum of the upper parabola and its value at the minimum of the lower one, as indicated in Fig. 1a and b. If $S_{\mathrm{HR}}$ is very small, i.e., the two potential energy surfaces are hardly shifted relative to each other, the vibrational wavefunction overlap will be small. If, additionally, the transition energy approximated by the energy difference between the two minima of the parabolas, $\Delta E$, is large, the barrier for the recombination process will be large (Fig. 1a), which is known as the 'energy gap law'.32,33,41,42 From this, it is immediately clear that for band-to-band recombination in high-quality inorganic semiconductors, due to the small associated Stokes shift and, hence, $S_{\mathrm{HR}}$, band-to-band recombination via the emission of multiple phonons is highly unlikely to occur. Conversely, when the two parabolas are massively shifted (Fig. 1b) and the wavefunction overlap is large, the limit $S_{\mathrm{HR}} E_{\mathrm{ph}}=\Delta E$ is approached, where $E_{\mathrm{ph}}=\hbar \omega$ is the energy for a phonon with frequency $\omega$. In this limit, the transition rate is maximized. Note that the Huang-Rhys factor is not a material constant, but depends on microscopic details of the states involved in the transition, and therefore varies between different scenarios.

Transition rate calculations according to Fig. 1 lead to a high temperature limit, in which the transition rate is thermally activated according to an activation energy, and a low temperature limit with a weak temperature dependence. Interestingly, this low temperature limit for the rate already shows a strong dependence on the number $p$ of phonons needed for a given transition ( $\left.p=\Delta E / E_{\mathrm{ph}}\right)$ and on the value of the Huang-Rhys factor $S_{\mathrm{HR}}$. To see that this is a general physical effect, consider the multiphonon transition rate $k$ in a semi-classical approximation valid over the whole temperature range, which has been derived by Markvart ${ }^{29}$

$$
k \propto \frac{\sqrt{2 \pi}}{\mathrm{h}^{2} \omega \sqrt{p \sqrt{1+x^{2}}}} \exp \left[p\left(\frac{\mathrm{h} \omega}{2 k T}+\sqrt{1+x^{2}}-x \cosh \left(\frac{\mathrm{h} \omega}{2 k T}\right)-\ln \left(\frac{1+\sqrt{1+x^{2}}}{x}\right)\right)\right] .
$$


Here, $T$ is temperature, $k$ the Boltzmann and $\mathrm{h}$ the reduced Planck constant.The parameter $x$ includes the dependence of the recombination rate on the ratio $p / S_{\mathrm{HR}}$ and is defined as ${ }^{29}$

$$
x=\left\{\begin{array}{l}
\frac{S_{\mathrm{HR}}}{p \sinh (\mathrm{h} \omega / 2 k T)} \text { for } S_{\mathrm{HR}}<p \\
\frac{p}{S_{\mathrm{HR}} \sinh (\mathrm{h} \omega / 2 k T)} \text { for } S_{\mathrm{HR}}>p
\end{array} .\right.
$$

Following the derivations outlined by Ridley ${ }^{43,44}$, it is possible to express the Huang-Rhys factor for polar coupling as

$$
S=\frac{3}{2(\mathrm{~h} \omega)^{2}}\left\{\frac{q^{2}\left(M_{\mathrm{r}} / V_{0}\right) \mathrm{h} \omega^{2}}{M_{\mathrm{r}} \omega q_{\mathrm{D}}^{2}}\left(\frac{1}{\varepsilon_{\infty}}-\frac{1}{\varepsilon}\right)\right\} I\left(-2,2 \mu, q_{\mathrm{D}} a^{*} v / 2\right)
$$

where $M_{\mathrm{r}}$ is the reduced mass, $q_{\mathrm{D}}$ is the radius of a sphere with the Brillouin-zone volume, $\varepsilon_{\infty}$ and $\varepsilon$ are the high- and low-frequency limit of the dielectric function, and $a^{*} v / 2$ is a rough estimate for the radius of the defect wavefunction in the 'quantum defect model'. The function $I$ is defined via

$$
I(a, b, c)=\frac{1}{(b c)^{2}} \int_{0}^{1} \frac{x^{a} \sin ^{2}\left(b \tan ^{-1}(c x)\right)}{\left[1+(c x)^{2}\right]^{b}}
$$

From Eq. (2) it can be seen that the number of phonons and the value of $S_{\mathrm{HR}}$ strongly influence the multiphonon transition rate. In fact, one finds that for $T \rightarrow 0, k \sim 1 / p$ !. Eqs. (3) and (4) use a series of parameters given in Table I for the calculations used to produce the data shown below. The energy of the defect level, i.e., the trap depth, $\Delta E$, determines the function $I$, and thus $S_{\mathrm{HR}}$, via the parameter $v=q\left(8 \pi \varepsilon a^{*} \Delta E\right)^{-1 / 2}$. The charge state of the trap also enters the calculation of both through the parameter $\mu$ : $\mu=\nu$ for positively charged defects, $\mu=0$ for neutral defects, and $\mu=-v$ for negatively charged defects. Therefore, the quantum defect model we use here cannot account for all possibly important microscopic details and effects, but it does include the important dependencies of the transition rate on the energy and charge state of the defect. 
For the prototypical MHP crystal $\mathrm{CH}_{3} \mathrm{NH}_{3} \mathrm{PbI}_{3}\left(\mathrm{MAPbI}_{3}\right)$ the phonon energies, the effective mass, and the reduced mass $M_{\mathrm{r}}$ are known (see Table I and ref. ${ }^{45}$ ). In particular, the vibrational energies of MHPs such as $\mathrm{MAPbI}_{3}$ have been reported from computations as well as infrared (IR) and Raman spectroscopy. ${ }^{45-51}$ These studies have shown that the longitudinal optical (LO) phonons, corresponding to Pb-halide modes, are relatively low in energy, which is consistent with the mechanically soft lattice of MHPs. ${ }^{52,53}$ We mention that in Eq. (3) it is assumed that the interaction is fully-screened via the "Pekar factor", defined as $\gamma=\varepsilon_{\infty}^{-1}-\varepsilon^{-1}$. However, it is a priori unclear which frequency range of $\varepsilon(f)$ is appropriate for describing polar coupling for a given transition. In absence of further information, we chose the experimentally-reported high- and low-frequency limit of $\varepsilon(f)$ (see Table 1 ), which maximizes polar coupling and, as discussed in detail below, counteracts the effect of low phonon energies we describe here. Therefore, we may now proceed to calculate $S_{\mathrm{HR}}$ for polar coupling based on Eqs. (3) and (4).

Fig. 2a shows the result for $\mathrm{MAPbI}_{3}$ as a function of the energy difference $\Delta E$ for the case of a positively charged, negatively charged, and neutral defect. $S_{\mathrm{HR}}$ is quite high due to the above-mentioned low phonon energy, $E_{\mathrm{ph}}=\hbar \omega=16.5 \mathrm{meV}$ (LO phonon), and the high "Pekar factor”, defined as $\gamma=\varepsilon_{\infty}^{-1}-\varepsilon^{-1}$. In addition, $S_{\mathrm{HR}}$ increases for larger values of $\Delta E$ with the exception of very shallow negatively charged defects. Note that such large $S_{\mathrm{HR}}$ values are not unprecedented ${ }^{54}$ and not in conflict with recently reported, much smaller values for similar perovskite systems either, ${ }^{55-57}$ as these experiments studied band-to-band transitions. We however are focussing on transitions between delocalized and localized states; the latter typically involve larger structural changes and larger values of $S_{\mathrm{HR}}$. Also note that the radius of the defect wavefunction enters our computation via the parameter $a^{*} v / 2$, as defined above.

Recombination via singly charged defects in semiconductors is typically described using Shockley-Read-Hall (SRH) statistics ${ }^{36,37}$ with a recombination rate given by 


$$
R_{\mathrm{SRH}}=\frac{n p-n_{0} p_{0}}{\left(n+n_{1}\right) \tau_{p}+\left(p+p_{1}\right) \tau_{n}}
$$

with the SRH lifetimes defined by $\tau_{n, p}=\left(k_{\mathrm{n}, \mathrm{p}} N_{t}\right)^{-1}$ and using the abbreviations $n_{1}=N_{\mathrm{C}} \exp \left[\left(E_{\mathrm{T}}-E_{C}\right) / k T\right]$ and $p_{1}=N_{\mathrm{V}} \exp \left[\left(E_{\mathrm{V}}-E_{\mathrm{T}}\right) / k T\right]$. Here, $n$ is the electron concentration, $p$ the hole concentration, the index ' 0 ' represents the equilibrium concentrations, $N_{\mathrm{T}}$ is the trap density, $N_{\mathrm{C}}$ is the conduction band effective density of states, $N_{\mathrm{V}}$ is the valence band effective density of states, $E_{\mathrm{C}}$ is the conduction band edge, $E_{\mathrm{V}}$ the valence band edge, $E_{\mathrm{T}}$ the trap position and $k_{\mathrm{n}, \mathrm{p}}$ is the recombination coefficient for recombination of an electron with a trapped hole or a hole with a trapped electron. Using the prefactors for the recombination rate as given by Ridley and discussed in the supporting information (SI), we obtain an estimate of $k_{\mathrm{n}}$ and $k_{\mathrm{p}}$ based on Eq. (1), the transition rate associated with a donor and acceptor like trap, respectively (see Fig. 2c). This estimate leads to rates that decrease strongly for higher energy differences between band and trap state. As illustrated in Fig. 2b, recombination via traps always involves two transitions, conduction band to trap and trap to valence band. Thus, $\max \left(E_{\mathrm{C}}-E_{\mathrm{T}}, E_{\mathrm{T}}-E_{\mathrm{V}}\right)$ will dominate the rate and midgap traps will be most detrimental because $\max \left(E_{\mathrm{C}}-E_{\mathrm{T}}, E_{\mathrm{T}}-E_{\mathrm{V}}\right)$ is minimized. For deep traps and forward bias, $n p>$ $n_{0} p_{0}, n_{1}<<n$, and $p_{1}<<$. Thus, the SRH recombination rate simplifies to $R_{\mathrm{SRH}} \approx n p /\left(n \tau_{p}+p \tau_{n}\right)$. If we additionally assume that we are in high-level injection (doping level is negligible relative to the optically or electrically injected charge carrier density), i.e., $n$ $=p$, we can write $R_{\mathrm{SRH}} \approx n /\left(\tau_{p}+\tau_{n}\right) \equiv n / \tau_{\text {eff }}$. We know that effective lifetimes $\tau_{\text {eff }}$ of MAPI have been measured to be on the order of $1 \mu$ s., ${ }^{8,58}$ Thus, we may now calculate the trap density needed to achieve a certain effective lifetime as $N_{\mathrm{T}} \approx\left(\left(\tau_{\mathrm{eff}} k_{\mathrm{n}}\right)^{-1}+\left(\tau_{\text {eff }} k_{\mathrm{p}}\right)^{-1}\right)^{-1}$. We also include the effect of Coulomb attraction between positively charged trap states and electrons and negatively charged trap states and holes via the so-called Sommerfeld factors, $s_{\mathrm{a}}$, as described in the SI. 
The results for the case of a donor like and an acceptor like trap are shown in Fig. 2c as a function of the trap energy, $E_{\mathrm{T}}$. Since the total rate will be limited by the slower of the two transitions, the resulting trap density required for a lifetime of $1 \mu$ s is symmetric with respect to midgap trap energies $\left(\sim 0.8 \mathrm{eV}\right.$ for $\left.\mathrm{MAPbI}_{3}\right)$ and it increases sharply when the trap is energetically no longer at midgap. This is due to the strong dependence of the recombination rate, as approximately given by Eq. (1), on the number of phonons involved in the transition, in which a larger number of phonons leads to much slower rates. For $\mathrm{MAPbI}_{3}$, due to the small optical phonon energy, a trap at midgap energy would require 49 phonons to be emitted instantaneously, making non-radiative recombination unlikely, which automatically results in the long lifetimes that were observed experimentally.

To put this finding into perspective, we mention that recently ${ }^{41}$ the high energy intramolecular vibrations in organic molecules have been shown to be one main reason for high losses due to non-radiative recombination in organic solar cells. In particular, the unavoidable $\mathrm{C}=\mathrm{C}$ double bond with a vibrational energy of $\sim 160 \mathrm{meV}$, as present in the backbone of any conjugated polymer or small molecule, was identified as the main culprit. The key observation of ref ${ }^{41}$ was that non-radiative voltage losses in organic solar cells follow the so-called energy gap law, ${ }^{32,33,42}$ which states that the recombination rate decreases with $\Delta E$. The energy gap law can be assessed from Eq. (1) and, given that $\Delta E$ does not directly enter Eq. (1) but instead the ratio $p=\Delta E / E_{\mathrm{ph}}$, one might conclude that lower phonon energies $E_{\mathrm{ph}}$ would be key to achieving low non-radiative voltage losses. As shown in the SI, the product of phonon energy and reduced ionic masses only varies mildly over a wide range of relevant semiconductors. Therefore, the beneficial effect of low phonon energies would imply that semiconductors made of heavier atoms show advantages in terms of a lower likelihood of non-radiative recombination via multiphonon emission. We note that this expectation is reminiscent of vibrational transitions in molecules, where it was shown that 
deuterides, due to the heavier mass of the atomic oscillator, relax much slower than corresponding hydrides. ${ }^{33,59,60}$

To calculate the effect of this on non-radiative lifetimes in MHPs, we first note that the phonon energy also enters the equation for the Huang-Rhys factor (Eq. (3)), with higher phonon energies leading to lower Huang-Rhys factors. Thus, the effects on $p$ and $S_{\mathrm{HR}}$ are partly cancelling in the evaluation of the rate (Eq. (1)). In the following, we keep the parameters for $\mathrm{MAPbI}_{3}$ (see Table I) constant, and vary the reduced mass and phonon energy such that their product remains constant (see SI) to then recalculate $S_{\mathrm{HR}}$ and the transition rates. Fig. $3 a$ shows that, as expected, $S_{\mathrm{HR}}$ strongly depends on phonon energy (and reduced mass) and decreases with higher phonon energies. In analogy to Fig. 2b, Fig. 3b shows the defect densities required for obtaining the generally accepted SRH lifetime of $\sim 1 \mu$ for $\mathrm{MAPbI}_{3}$, when varying the phonon energy (and reduced mass) of the atomic oscillator. For higher phonon energies, the necessary defect density at midgap energies for obtaining the experimentally determined lifetime already decreases, i.e. lower phonon energies make multiphonon emission less likely.

Remarkably, however, the strongest effect is observed for defect levels away from midgap energy, which are the most relevant energies for practical MHP cells. Figure 3b shows that the necessary defect density to achieve a certain lifetime is much less sensitive to the defect energy position at high phonon energies than it is for lower ones. In the extreme situation of a large phonon energy of $160 \mathrm{meV}$, which mimics organic semiconductors, the position of the defect level becomes even somewhat unimportant, as non-radiative band-toband transitions, which are very unlikely in inorganic semiconductors, become possible. This finding implies that low-energy phonons, for which the required defect density away from the midgap energy strongly increases, help suppress non-radiative recombination via harmful defects deep in the gap. This is also in line with the observation that ideality factors $\sim 1$ are common in fully depleted organic solar cells, a situation that is typically not encountered in 
fully depleted inorganic solar cells. ${ }^{39,61}$ It may also explain why harmful defects ${ }^{62-65}$ and higher ideality factors, close to two, are sometimes observed ${ }^{66-68}$ in organic solar cells without massively deteriorating photovoltaic performance.

Thus, we can readily conclude that for recombination to be fast in a polar semiconductor with low phonon energies, not only the defect density must be high but, in addition, the position of the defect must be very close to midgap. Shifts of the state energy relative to the midgap energy will increase the number of phonons needed for the larger energy of the two transitions in the scenario discussed above, such that the total recombination rate will decrease substantially. This effect is much less pronounced for higher phonon energies as is the case in typical inorganic semiconductors, in which a smaller number of phonons is required for a single transition. Based on the multiphonon transition scenario, our data and analysis imply that a low density of deep defects (but, potentially, a high density of shallow defects) in a material with low phonon energies leads to low non-radiative recombination rates and, thus, long carrier lifetimes. In view of recent proposals for defect tolerance and healing in MHPs, ${ }^{69,70}$ one may adapt the view that these phenomena are driven in part by the low-energy phonons of MHPs, which strongly reduce the detrimental impact of deep defects and associated non-radiative recombination. From this, the low phonon energies of MHPs emerge as an important 'microscopic materials design' parameter for further optimizing perovskite solar cells.

We consider this result as an important step towards establishing a deeper understanding of how recombination kinetics in addition to defect densities contribute to long charge-carrier lifetimes in perovskite solar cells. However, we would like to point out that models for multiphonon processes in semiconductors have a high level of complexity. In particular, the series of exponential terms in Eq. (1) leads to large changes in the result for small deviations in input parameters. In addition, strictly speaking the equations are still only approximations to an even more complex physical reality. For example, theoretical calculations showed that 
dephasing times in MPHs depend on their ionic composition and doping, ${ }^{71}$ which influences electronic transitions, but is not considered in our model. In the following, we will therefore briefly discuss further potential shortcomings of the model as well as further steps for model development and experimental validation.

One of the key assumptions for the calculations presented in Figs. 2 and 3 is that polar coupling dominates multiphonon transitions. This assumption was made based on the high Pekar factor, $\gamma=\varepsilon_{\infty}^{-1}-\varepsilon^{-1}$, in $\mathrm{MAPbI}_{3}$. For less polar semiconductors, deformation potential coupling due to optical phonons might be the dominant mechanism. For $\mathrm{MAPbI}_{3}$, the effect of polar coupling, especially for different frequencies of the dielectric response, has not been systematically compared to the effect of deformation potential coupling due to optical phonons. While Ridley proposed an equation to also calculate $S_{\mathrm{HR}}$ for deformation potential coupling, the actual calculation requires knowledge on the so-called optical deformation potential constant. This quantity is rather difficult to measure experimentally or calculate theoretically, and is currently unknown for $\mathrm{MAPbI}_{3}$. Therefore, the deformation potential constant has in the past typically been used as an 'adjustable constant'. ${ }^{72}$ In the SI, we therefore show the recombination rates for different values of the optical deformation potential constant. These data show that larger values of the optical deformation potential constant reduce the beneficial effect of low phonon energies on recombination, which means that our findings do not hold for all types of semiconductors. Within our model and parameters, we determine an upper limit for the optical deformation constant of $\sim 5 \times 10^{8}$ $\mathrm{eV} / \mathrm{cm}$ for which a noticeable positive effect of the low phonon energy on recombination is still observed.

Furthermore, the theoretical approach discussed above makes very specific assumptions on the vibrations involved in multiphonon recombination: in $\mathrm{MAPbI}_{3}$, high-energy vibrations are associated with the organic molecule. However, the electronic states of the organic cation 
are energetically far-off the frontier valence and conduction band states, which are determined by contributions from $\mathrm{Pb}$ and I atoms. Non-adiabatic molecular-dynamics computations have also shown that the Pb-halide stretching and bending modes couple strongly to the electronic structure, and are thus important factors determining the recombination rates at $\mathrm{MAPbI}_{3} / \mathrm{TiO}_{2}$ interfaces. ${ }^{71,73}$ Therefore, in our single mode model we have chosen the highest-energy longitudinal optical phonon associated with Pb-I vibrations. ${ }^{45}$ Thus, the model does not explicitly include information about multiple phonons, with individual frequencies and coupling constants, and their effect on multiphonon transitions, i.e., the phonon density of states is disregarded. We note that a fully predictive model, including first-principles data of all the relevant modes and their coupling, ${ }^{71,73}$ is far from trivial but conceivable in principle. ${ }^{74,75}$ In practice, MHPs such as $\mathrm{MAPbI}_{3}$ exhibit strong anharmonic vibrational contributions at room temperature, ${ }^{45,50}$ complicated photo-excited carrier relaxation effects, ${ }^{76,77}$ and intriguing decoherence effects on electronic transitions. ${ }^{71}$ While these effects go far beyond the scope of our purely model-based approach, elucidating their impact on carrier lifetimes indeed motivates future studies. In the same spirit, a further general question about the validity of multiphonon transition theory is the inclusion of the effect of entropy. ${ }^{78,79}$ For higher numbers of phonons needed for a given transition, the number of contributing possible combinations of phonon modes becomes increasingly larger. This effect gives rise to the Meyer-Neldel rule. The very general question how to merge theories of multiphonon recombination with entropy considerations is discussed in ref. ${ }^{78}$ and currently still under debate. ${ }^{79}$

We also would like to put our finding that low phonon energies are key to reducing nonradiative recombination into perspective of other currently discussed effects explaining the lifetime of carriers in MHPs cells. First, several studies reported the impact of the indirect band gap $22,23,25,80$ and polar organic molecule ${ }^{81,82}$ on radiative and non-radiative lifetimes of MHPs. Interestingly, a recent terahertz spectroscopy study ${ }^{83}$ on the all-inorganic MHP CsPbI 3 
showed that systems lacking a polar cation can still exhibit similar bimolecular recombination rates as hybrid MHPs, which indicates that the polar cation does not strongly impact radiative transitions. The role of the polar organic cation is also discussed in the context of large polaron formation MHPs cells. ${ }^{27,28}$ An interesting consequence of the theory of multiphonon recombination with polar coupling is that high Pekar factors, $\gamma=\varepsilon_{\infty}^{-1}-\varepsilon^{-1}$, in general lead to stronger non-radiative recombination, as they enhance $S_{\mathrm{HR}}$ (see Eqs. (1)-(4)). This suggests that stronger polaronic effects, and higher values of $\gamma$, would not lead to slower band-to-band or defect-to-band recombination as is currently speculated. Note that this explanation is not questioning the formation of large polarons in MHPs per se. Rather, we here address the issue whether recombination is dictated by two (possibly screened) charges finding each other in a diffusion-limited recombination process, or by the time required to emit $\sim 50$ phonons. We tentatively conclude that the rate for multiphonon transition is the more relevant factor in limiting the lifetime, based on two observations: In organic photovoltaics, with materials that show orders of magnitude lower mobilities compared to MHPs, there is solid evidence for the effect of the energy gap law, ${ }^{41}$ and hence phonon energies. This suggests that even for this case, Langevin-type recombination is not limiting the total recombination rate. In MHPs, Langevin-type recombination models indeed overestimate the recombination rates by orders of magnitude. From this, we conclude that the long lifetime in MHPs is likely not observed because of the high $\gamma=\varepsilon_{\infty}^{-1}-\varepsilon^{-1}$, but despite the high $\gamma=\varepsilon_{\infty}^{-1}-\varepsilon^{-1}$.

Finally, we comment on experimental determination of capture cross sections in perovskite solar cells as a way of testing our conclusions. In order to determine $S_{\mathrm{HR}}$ for a specific defect-mediated transition one measures the temperature dependence of the capture cross section in the high temperature region of Eq. (1), where an activation energy becomes visible. This activation energy $E_{\mathrm{B}}=\left(\Delta E-S_{\mathrm{HR}} \mathrm{h} \omega\right)^{2} /\left(4 S_{\mathrm{HR}} \mathrm{h} \omega\right)$ solely depends on the trap depth, $S_{\mathrm{HR}}$, and the phonon energy, which in principle allows for extraction of the Huang- 
Rhys factor if $S_{\mathrm{HR}}$ is the only unknown. Last but far from least, we mention that such studies would be interesting especially for MHPs, where the ionic mass can be changed by ionic composition, e.g., by using $\mathrm{Br}$ instead of I, Sn instead of $\mathrm{Pb}$, and different A-site cations. Such substitutions will change the ionic masses, thereby the optical phonon energies, which in light of our results and shown in the SI is expected to impact recombination rates and lifetimes.

In summary, we have used model calculations to examine the effect of low-energy phonons on charge-carrier lifetimes of MHPs. Transitions between localized defect states and delocalized states were studied in a multiphonon transition scenario including the effect of defect energy and densities. We found that the long charge carrier lifetimes of $\mathrm{MAPbI}_{3}$ can be explained in this scenario and showed that low-energy phonons are key towards achieving these lifetimes: In polar semiconductors with low-energy phonons, non-radiative recombination via deep traps is suppressed by many orders of magnitudes when compared to semiconductors with higher phonon energies, which can readily be deduced from the physics of multiphonon transitions. Here, we found that this effect becomes considerably more pronounced if the defect energy is some few $100 \mathrm{meV}$ away from the midgap energy, i.e., the impact of low phonon energies on non-radiative transitions is especially pronounced for the practically most relevant defect scenarios. Based on our findings, we conclude that even a relatively high density of deep defects is compatible with a low non-radiative recombination rate in a polar semiconductor with low phonon energies, especially if the defect energies are not directly at midgap. With this, the low phonon energies of MHPs emerge as an important microscopic material parameter that together with shallow trap energies could represent one important ingredient for the success of MHPs. This aspect needs to be taken into account when aiming to further advance perovskite solar cells. 
Author Information

Corresponding Author:

*To whom correspondence should be addressed. E-Mail: t.kirchartz@fz-juelich.de, david.egger@physik.uni-regensburg.de

\section{Acknowledgements}

TK and UR acknowledge support from the DFG (Grant Nos. KI-1571/2-1 and RA 473/7-1). TM acknowledges support via the Centre for Advanced Photovoltaics supported by the Czech Ministry of Education, Youth and Sport (CZ.02.1.01/0.0/0.0/15_003/0000464). DAE acknowledges funding provided by the Alexander von Humboldt Foundation in the framework of the Sofja Kovalevskaja Award endowed by the German Federal Ministry of Education and Research. The authors would like to thank Vanessa Wood, Nuri Yazdani (both ETH Zürich), David Cahen, Omer Yaffe (both Weizmann Institute of Science), and Deniz Bozyigit (Battrion) for fruitful discussions.

\section{Supporting Information Available}

Explanation for the choice of the prefactor for the multiphonon transition rate. Sommerfeld factors to take Coulomb attraction into account. Temperature dependence of the rates. Dependence of reduced mass on phonon energy. Application of the methodology to $\mathrm{CH}_{3} \mathrm{NH}_{3} \mathrm{PbBr}_{3}$ and comparison to $\mathrm{CH}_{3} \mathrm{NH}_{3} \mathrm{PbI}_{3}$. Influence of deformation coupling. 

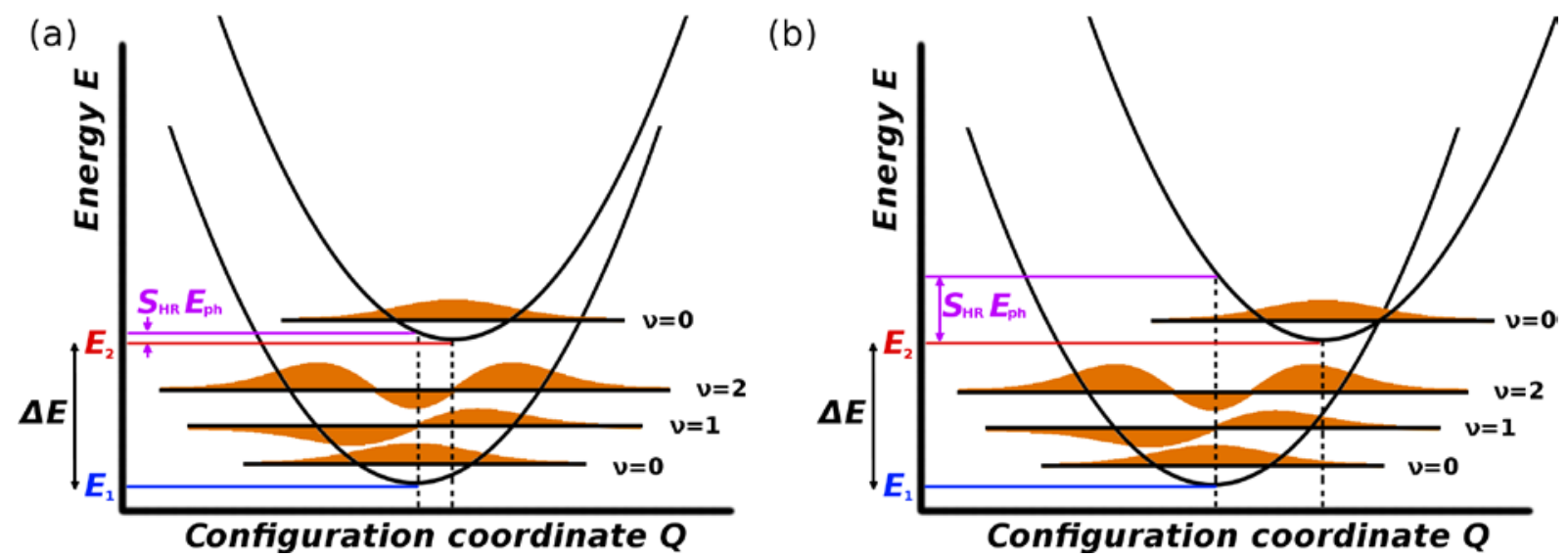

Figure 1: Schematic showing a configuration coordinate diagram with small (a) and large (b) Huang-Rhys factor, $S_{\mathrm{HR}} . E_{1}$ and $E_{2}$ indicate the minimum energies of two representative potential energy surfaces that correspond to the two states in the multiphonon transition scenario, which are separated by an energy difference, $\Delta E . E_{\mathrm{ph}}$ denotes the energy of the optical phonon emitted in the multiphonon process and $v$ is the vibrational quantum number. 


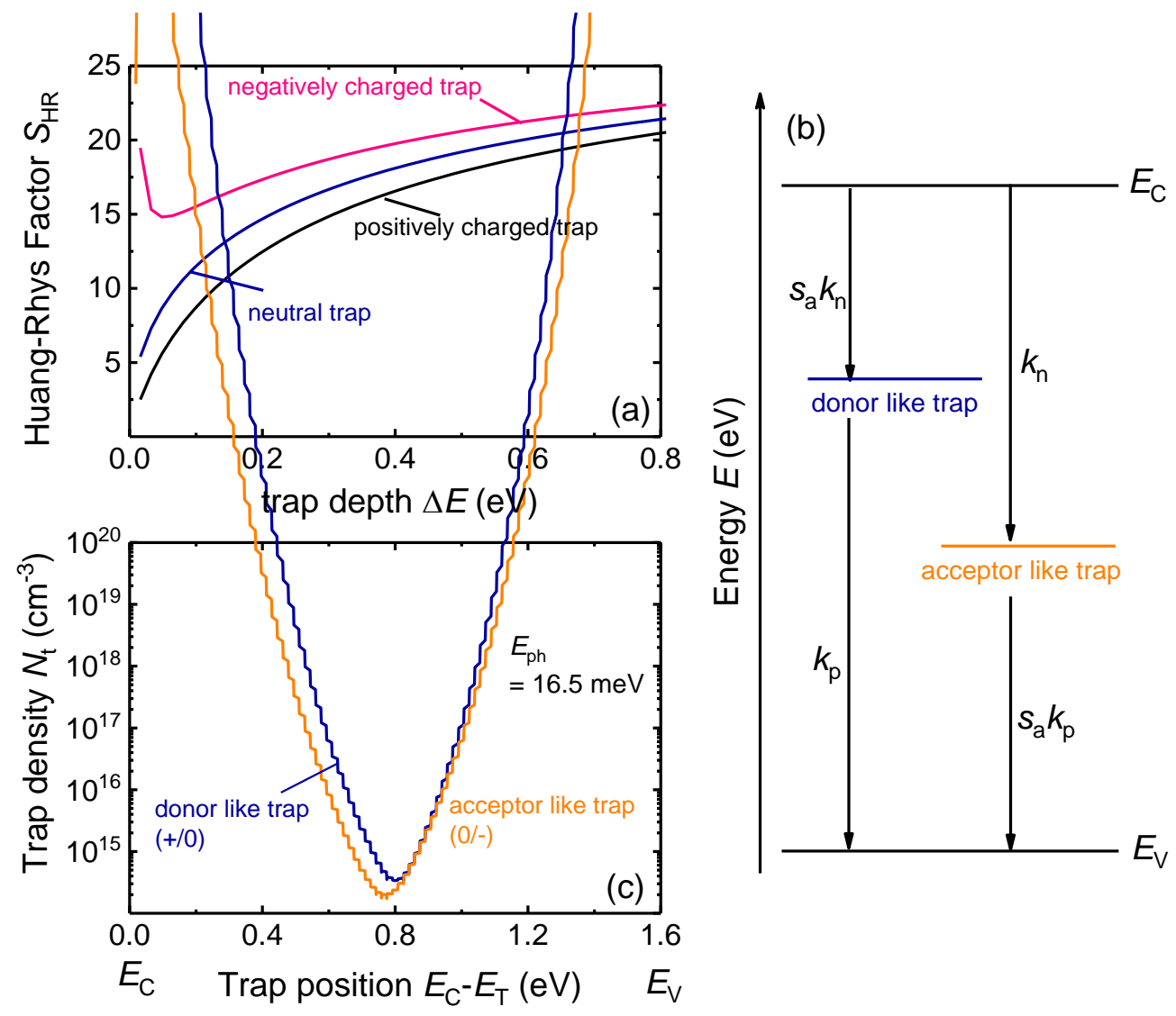

Figure 2: (a) Huang-Rhys factor as a function of the energy difference $\Delta E$, as defined in Fig. 1, using the parameters given in Table I. The result changes with the charge state of the trap according to Eqs. (3) and (4). (b) Schematic of the transition from the conduction band to a donor and acceptor like trap, and from a donor and an acceptor like trap to the valence band. The respective rates, $k$, and the Sommerfeld factor, $s_{\mathrm{a}}$, are indicated (see text for details). (c) Trap density needed to achieve a SRH lifetime of $1 \mu$ s for a phonon energy of $16.5 \mathrm{meV}$ as given by ref. ${ }^{45}$. 

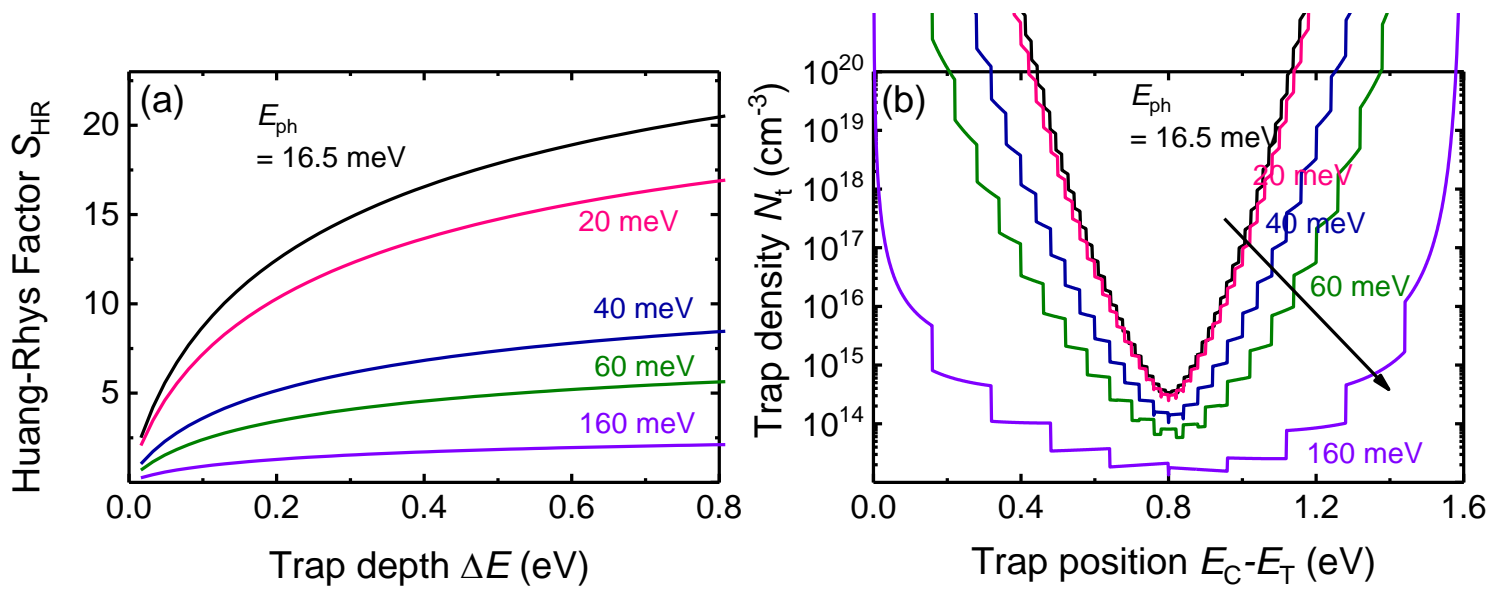

Figure 3: (a) Huang-Rhys factor for a positively charged trap as a function of $\Delta E$ and the phonon energy. All parameters were kept as defined in Table I, however, the product $M_{\mathrm{r}} E_{\mathrm{ph}}$ was kept constant (see SI). (b) Trap density needed to achieve a SRH lifetime of $1 \mu \mathrm{s}$, for phonon energies ranging from $16.5 \mathrm{meV}$ to $160 \mathrm{meV}$, showing that larger phonon energies make the exact position of the trap less important with midgap energy always remaining the worst-case scenario. 
Table I: Parameter and abbreviations used in the simulations. The simulations were done assuming a temperature $T=300 \mathrm{~K}$.

\begin{tabular}{lll}
\hline Parameter & Symbol or Equation & Value \\
\hline phonon energy & $E_{\mathrm{ph}}=\hbar \omega$ & $16.5 \mathrm{meV}$ (LO Phonon $)^{45}$ \\
reduced mass & $M_{\mathrm{r}}=M_{\mathrm{Pb}} M_{\mathrm{I}} /\left(M_{\mathrm{Pb}}+M_{\mathrm{I}}\right)$ & 78.7 \\
permittivity(frequency) & $\varepsilon(f)$ & $\varepsilon(0)=33.5 \varepsilon_{0}$ \\
& & $\varepsilon(\infty)=5.0 \varepsilon_{0}{ }^{45}$ \\
lattice constant & $a_{0}$ & $6.3 \AA^{84}$ \\
radius of sphere with & $q_{\mathrm{D}}=\left(6 \pi^{2}\right)^{1 / 3} / a_{0}$ & $6.2 \mathrm{~nm}^{-1}$ \\
Brillouin zone volume & $m_{\mathrm{eff}}$ & $0.2(\mathrm{assumed}$ equal for \\
effective mass & & electrons and holes) \\
Bohr radius & $a_{\mathrm{H}}=4 \pi \varepsilon_{0} \mathrm{~h}^{2} / \mathrm{mq}^{2}$ & $5.292 \times 10^{-2} \mathrm{~nm}$ \\
effective Bohr radius & $a^{*}=a_{\mathrm{H}} \varepsilon_{\mathrm{r}}(0) / m_{\mathrm{eff}}$ & $8.9 \mathrm{~nm}$ \\
Rydberg energy & $R_{\mathrm{H}}=q^{2} /\left(8 \pi \varepsilon_{0} a_{H}\right)$ & $13.605 \mathrm{eV}$ \\
effective Rydberg energy & $R^{*}=q^{2} /(8 \pi \varepsilon(0) a *)$ & $2.4 \mathrm{meV}$ \\
& $v=q(8 \pi \varepsilon(0) a * \Delta E)^{-1 / 2}$ & variable \\
Sommerfeld factor ${ }^{85}$ & $S_{\mathrm{a}}=4\left(\pi R^{*} / k T\right)^{1 / 2}$ & 2.2 \\
\hline
\end{tabular}


References

(1) Correa-Baena, J. P.; Saliba, M.; Buonassisi, T.; Grätzel, M.; Abate, A.; Tress, W.; Hagfeldt, A. Promises and Challenges of Perovskite Solar Cells. Science 2017, 358, 739.

(2) Bi, D.; Tress, W.; Dar, M. I.; Gao, P.; Luo, J.; Renevier, C.; Schenk, K.; Abate, A.; Giordano, F.; Correa Baena, J. P. et al. Efficient Luminescent Solar Cells Based on Tailored Mixed-Cation Perovskites. Sci Adv 2016, 2, e1501170.

(3) Saliba, M.; Matsui, T.; Domanski, K.; Seo, J. Y.; Ummadisingu, A.; Zakeeruddin, S. M.; Correa-Baena, J. P.; Tress, W. R.; Abate, A.; Hagfeldt, A. et al. Incorporation of Rubidium Cations into Perovskite Solar Cells Improves Photovoltaic Performance. Science 2016, 354, 206.

(4) Tress, W.; Marinova, N.; Inganas, O.; Nazeeruddin, M. K.; Zakeeruddin, S. M.; Graetzel, M. Predicting the Open-Circuit Voltage of $\mathrm{CH}_{3} \mathrm{NH}_{3} \mathrm{PbI}_{3}$ Perovskite Solar Cells Using Electroluminescence and Photovoltaic Quantum Efficiency Spectra: the Role of Radiative and Non-Radiative Recombination. Adv. Energy Mater. 2015, 5, 1400812.

(5) Tvingstedt, K.; Malinkiewicz, O.; Baumann, A.; Deibel, C.; Snaith, H. J.; Dyakonov, V.; Bolink, H. J. Radiative Efficiency of Lead Iodide Based Perovskite Solar Cells. Sci. Rep. 2014, 4, 6071.

(6) Stranks, S. D.; Eperon, G. E.; Grancini, G.; Menelaou, C.; Alcocer, M. J. P.; Leijtens, T.; Herz, L. M.; Petrozza, A.; Snaith, H. J. Electron-Hole Diffusion Lengths Exceeding 1 Micrometer in an Organometal Trihalide Perovskite Absorber. Science 2013, 342, 341-344.

(7) Bi, Y.; Hutter, E. M.; Fang, Y.; Dong, Q.; Huang, J.; Savenije, T. J. Charge Carrier Lifetimes Exceeding $15 \mu \mathrm{s}$ in Methylammonium Lead Iodide Single Crystals. J. Phys. Chem. Lett. 2016, 7, 923-928.

(8) deQuilettes, D. W.; Koch, S.; Burke, S.; Paranji, R. K.; Shropshire, A. J.; Ziffer, M. E.; Ginger, D. S. Photoluminescence Lifetimes Exceeding 8 ss and Quantum Yields Exceeding $30 \%$ in Hybrid Perovskite Thin Films by Ligand Passivation. ACS Energy Lett. 2016, 1, 438-444.

(9) deQuilettes, D. W.; Vorpahl, S. M.; Stranks, S. D.; Nagaoka, H.; Eperon, G. E.; Ziffer, M. E.; Snaith, H. J.; Ginger, D. S. Impact of Microstructure on Local Carrier Lifetime in Perovskite Solar Cells. Science 2015, 348, 683-686.

(10) deQuilettes, D. W.; Zhang, W.; Burlakov, V. M.; Graham, D. J.; Leijtens, T.; Osherov, A.; Bulovic, V.; Snaith, H. J.; Ginger, D. S.; Stranks, S. D. PhotoInduced Halide Redistribution in Organic-Inorganic Perovskite Films. Nat. Commun 2016, 7, 11683. 
(11) Brenner, T. M.; Egger, D. A.; Kronik, L.; Hodes, G.; Cahen, D. Hybrid OrganicInorganic Perovskites: Low-Cost Semiconductors With Intriguing ChargeTransport Properties. Nat. Rev. Mater. 2016, 1, 15007.

(12) Jeon, N. J.; Noh, J. H.; Yang, W. S.; Kim, Y. C.; Ryu, S.; Seo, J.; Seok, S. I. Compositional Engineering of Perovskite Materials for High-Performance Solar Cells. Nature 2015, 517, 476-480.

(13) Yang, W. S.; Noh, J. H.; Jeon, N. J.; Kim, Y. C.; Ryu, S.; Seo, J.; Seok, S. I. HighPerformance Photovoltaic Perovskite Layers Fabricated Through Intramolecular Exchange. Science 2015, 348, 1234-1237.

(14) Bush, K. A.; Palmstrom, A. F.; Yu, Z. J.; Boccard, M.; Cheacharoen, R.; Mailoa, J. P.; McMeekin, D. P.; Hoye, R. L. Z.; Bailie, C. D.; Leijtens, T. et al. 23.6\%Efficient Monolithic Perovskite/Silicon Tandem Solar Cells With Improved Stability. Nat. Energy 2017, 2, 17009.

(15) Saliba, M.; Orlandi, S.; Matsui, T.; Aghazada, S.; Cavazzini, M.; Correa-Baena, J. P.; Gao, P.; Scopelliti, R.; Mosconi, E.; Dahmen, K. H. A Molecularly Engineered Hole-Transporting Material for Efficient Perovskite Solar Cells. Nat. Energy 2016, 15017.

(16) Leijtens, T.; Eperon, G. E.; Noel, N. K.; Habisreutinger, S. N.; Petrozza, A.; Snaith, H. J. Stability of Metal Halide Perovskite Solar Cells. Adv. Energy Mater. 2015, 5, 1500963-1500n/a.

(17) Rolston, N.; Printz, A. D.; Tracy, J. M.; Weerasinghe, H. C.; Vak, D.; Haur, L. J.; Priyadarshi, A.; Mathews, N.; Slotcavage, D. J.; McGehee, M. D. et al. Effect of Cation Composition on the Mechanical Stability of Perovskite Solar Cells. Adv. Energy Mater. 2017, 1702116-1702n/a.

(18) Brandt, R. E.; Stevanoviç, V.; Ginley, D. S.; Buonassisi, T. Identifying DefectTolerant Semiconductors With High Minority-Carrier Lifetimes: Beyond Hybrid Lead Halide Perovskites. MRS Communications 2015, 5, 265-275.

(19) Yu, L.; Zunger, A. Identification of Potential Photovoltaic Absorbers Based on FirstPrinciples Spectroscopic Screening of Materials. Phys. Rev. Lett. 2012, 108, 068701.

(20) Blank, B.; Kirchartz, T.; Lany, S.; Rau, U. Selection Metric for Photovoltaic Materials Screening Based on Detailed-Balance Analysis. Phys. Rev. Applied 2017, 8, 024032.

(21) Yin, W. J.; Shi, T.; Yan, Y. Unusual Defect Physics in CH3NH3PbI3 Perovskite Solar Cell Absorber. Appl. Phys. Lett. 2014, 104, 063903.

(22) Hutter, E. M.; Gelvez-Rueda, M. C.; Osherov, A.; Bulovic, V.; Grozema, F. C.; Stranks, S. D.; Savenije, T. J. Direct-Indirect Character of the Bandgap in Methylammonium Lead Iodide Perovskite. Nat. Mater. 2017, 16, 115-120. 
(23) Wang, T.; Daiber, B.; Frost, J. M.; Mann, S. A.; Garnett, E. C.; Walsh, A.; Ehrler, B. Indirect to Direct Bandgap Transition in Methylammonium Lead Halide Perovskite. Energ. Environ. Sci. 2017, 10, 509-515.

(24) Kirchartz, T.; Rau, U. Decreasing Radiative Recombination Coefficients Via an Indirect Band Gap in Lead Halide Perovskites. The Journal of Physical Chemistry Letters 2017, 8, 1265-1271.

(25) Zheng, F.; Tan, L. Z.; Liu, S.; Rappe, A. M. Rashba Spin-Orbit Coupling Enhanced Carrier Lifetime in CH3NH3PbI3. Nano Lett. 2015, 15, 7794-7800.

(26) Zhou, Y.; Long, G. Low Density of Conduction and Valence Band States Contribute to the High Open-Circuit Voltage in Perovskite Solar Cells. The Journal of Physical Chemistry C 2017, 121, 1455-1462.

(27) Miyata, K.; Meggiolaro, D.; Trinh, M. T.; Joshi, P. P.; Mosconi, E.; Jones, S. C.; De Angelis, F.; Zhu, X. Y. Large Polarons in Lead Halide Perovskites. Sci Adv 2017, 3 .

(28) Miyata, K.; Atallah, T. L.; Zhu, X. Y. Lead Halide Perovskites: Crystal-Liquid Duality, Phonon Glass Electron Crystals, and Large Polaron Formation. Sci Adv 2017, 3.

(29) Markvart, T. Multiphonon recombination. In Recombination in Semiconductors, Landsberg, P. T., Ed.; Cambridge University Press: Cambridge, 2003; pp 467-468.

(30) Markvart, T. Semiclassical Theory of Non-Radiative Transitions. Journal of Physics C: Solid State Physics 1981, 14, L895.

(31) Huang, K.; Rhys, A. Theory of Light Absorption and Non-Radiative Transitions in F-Centres. Proc R Soc Lond A Math Phys Sci 1950, 204, 406.

(32) Bixon, M.; Jortner, J.; Cortes, J.; Heitele, H.; Michel-Beyerle, M. E. Energy Gap Law for Nonradiative and Radiative Charge Transfer in Isolated and in Solvated Supermolecules. The Journal of Physical Chemistry 1994, 98, 7289-7299.

(33) Nitzan, A.; Mukamel, S.; Jortner, J. Energy Gap Law for Vibrational Relaxation of a Molecule in a Dense Medium. The Journal of Chemical Physics 1975, 63, 200-207.

(34) Tiedje, T.; Yablonovitch, E.; Cody, G. D.; Brooks, B. G. Limiting Efficiency of Silicon Solar-Cells. IEEE Trans. Elec. Dev. 1984, 31, 711-716.

(35) Green, M. A. Limits on the Open-Circuit Voltage and Efficiency of Silicon SolarCells Imposed by Intrinsic Auger Processes. IEEE Trans. Elec. Dev. 1984, 31, 671-678.

(36) Shockley, W.; Read, W. T. Statistics of the Recombination of Holes and Electrons. Phys. Rev. 1952, 87, 835-842.

(37) Hall, R. N. Electron-Hole Recombination in Germanium. Phys. Rev. 1952, 87, 387. 
(38) Simmons, J. G.; Taylor, G. W. Nonequilibrium Steady State Statistics and Associated Effects for Insulators and Semiconductors Containing an Arbitrary Distribution of Traps. Phys. Rev. B 1971, 4, 502-511.

(39) Pieters, B. E.; Stiebig, H.; Zeman, M.; van Swaaij, R. A. C. M. Determination of the Mobility Gap of Intrinsic Mu C-Si:H in P-i-n Solar Cells. J. Appl. Phys. 2009, 105, 044502.

(40) Pieters, B. E. Characterization of Thin-Film Silicon Materials and Solar Cells through Numerical Modelling; PhD Thesis Delft University of Technology: Delft, 2008.

(41) Benduhn, J.; Tvingstedt, K.; Piersimoni, F.; Ullbrich, S.; Fan, Y.; Tropiano, M.; McGarry, K. A.; Zeika, O.; Riede, M. K.; Douglas, C. J. et al. Intrinsic NonRadiative Voltage Losses in Fullerene-Based Organic Solar Cells. Nat. Energy 2017, 2, 17053.

(42) Englman, R.; Jortner, J. The Energy Gap Law for Radiationless Transitions in Large Molecules. Mol. Phys. 1970, 18, 145-164.

(43) Ridley, B. K. On the Multiphonon Capture Rate in Semiconductors. Solid State Electron 1978, 21, 1319-1323.

(44) Ridley, B. K. Quantum Processes in Semiconductors; Oxford University Press: Oxford, 2013.

(45) Sendner, M.; Nayak, P. K.; Egger, D. A.; Beck, S.; Muller, C.; Epding, B.; Kowalsky, W.; Kronik, L.; Snaith, H. J.; Pucci, A. et al. Optical Phonons in Methylammonium Lead Halide Perovskites and Implications for Charge Transport. Mater. Horiz. 2016, 3, 613-620.

(46) Quarti, C.; Grancini, G.; Mosconi, E.; Bruno, P.; Ball, J. M.; Lee, M. M.; Snaith, H. J.; Petrozza, A.; Angelis, F. D. The Raman Spectrum of the $\mathrm{CH}_{3} \mathrm{NH}_{3} \mathrm{PbI}_{3}$ Hybrid Perovskite: Interplay of Theory and Experiment. The Journal of Physical Chemistry Letters 2014, 5, 279-284.

(47) Brivio, F.; Frost, J. M.; Skelton, J. M.; Jackson, A. J.; Weber, O. J.; Weller, M. T.; Goni, A. R.; Leguy, A. M. A.; Barnes, P. R. F.; Walsh, A. Lattice Dynamics and Vibrational Spectra of the Orthorhombic, Tetragonal, and Cubic Phases of Methylammonium Lead Iodide. Phys. Rev. B 2015, 92, 144308.

(48) Wright, A. D.; Verdi, C.; Milot, R. L.; Eperon, G. E.; Perez-Osorio, M. A.; Snaith, H. J.; Giustino, F.; Johnston, M. B.; Herz, L. M. Electron-Phonon Coupling in Hybrid Lead Halide Perovskites. Nat. Commun. 2016, 7, 11755.

(49) Perez-Osorio, M. A.; Milot, R. L.; Filip, M. R.; Patel, J. B.; Herz, L. M.; Johnston, M. B.; Giustino, F. Vibrational Properties of the Organic-Inorganic Halide Perovskite $\mathrm{CH}_{3} \mathrm{NH}_{3} \mathrm{PbI}_{3}$ From Theory and Experiment: Factor Group Analysis, First-Principles Calculations, and Low-Temperature Infrared Spectra. The Journal of Physical Chemistry C 2015, 119, 25703-25718. 
(50) Yaffe, O.; Guo, Y.; Tan, L. Z.; Egger, D. A.; Hull, T.; Stoumpos, C. C.; Zheng, F.; Heinz, T. F.; Kronik, L.; Kanatzidis, M. G. et al. Local Polar Fluctuations in Lead Halide Perovskite Crystals. Phys. Rev. Lett. 2017, 118, 136001.

(51) Mattoni, A.; Filippetti, A.; Saba, M. I.; Delugas, P. Methylammonium Rotational Dynamics in Lead Halide Perovskite by Classical Molecular Dynamics: The Role of Temperature. The Journal of Physical Chemistry C 2015, 119, 17421-17428.

(52) Rakita, Y.; Cohen, S. R.; Kedem, N. K.; Hodes, G.; Cahen, D. Mechanical Properties of $\mathrm{APbX}_{3}\left(\mathrm{~A}=\mathrm{Cs}\right.$ or $\mathrm{CH}_{3} \mathrm{NH}_{3} ; \mathrm{X}=\mathrm{I}$ or $\left.\mathrm{Br}\right)$ Perovskite Single Crystals. MRS Communications 2015, 5, 623-629.

(53) Sun, S.; Fang, Y.; Kieslich, G.; White, T. J.; Cheetham, A. K. Mechanical Properties of Organic-Inorganic Halide Perovskites, $\mathrm{CH}_{3} \mathrm{NH}_{3} \mathrm{PbX}_{3}(\mathrm{X}=\mathrm{I}, \mathrm{Br}$ and $\mathrm{Cl}$ ), by Nanoindentation. J. Mater. Chem. A 2015, 3, 18450-18455.

(54) Henry, C. H.; Lang, D. V. Nonradiative Capture and Recombination by Multiphonon Emission in GaAs and GaP. Phys. Rev. B 1977, 15, 989-1016.

(55) Brennan, M. C.; Herr, J. E.; Nguyen-Beck, T. S.; Zinna, J.; Draguta, S.; Rouvimov, S.; Parkhill, J.; Kuno, M. Origin of the Size-Dependent Stokes Shift in CsPbBr3 Perovskite Nanocrystals. J. Am. Chem. Soc. 2017, 139, 1220112208.

(56) Fu, M.; Tamarat, P.; Huang, H.; Even, J.; Rogach, A. L.; Lounis, B. Neutral and Charged Exciton Fine Structure in Single Lead Halide Perovskite Nanocrystals Revealed by Magneto-Optical Spectroscopy. Nano Lett. 2017, 17, 2895-2901.

(57) Iaru, C. M.; Geuchies, J. J.; Koenraad, P. M.; Vanmaekelbergh, D. l.; Silov, A. Y. Strong Carrier-Phonon Coupling in Lead Halide Perovskite Nanocrystals. Acs Nano 2017, 11, 11024-11030.

(58) Staub, F.; Hempel, H.; Hebig, J. C.; Mock, J.; Paetzold, U. W.; Rau, U.; Unold, T.; Kirchartz, T. Beyond Bulk Lifetimes: Insights into Lead Halide Perovskite Films From Time-Resolved Photoluminescence. Phys. Rev. Applied 2016, 6, 044017.

(59) Bondybey, V. E.; Brus, L. E. Interdependence of Guest Radiationless Transitions and Localized Phonon Structure: $\mathrm{NH}$ and $\mathrm{ND}\left(\mathrm{A}^{3}\right)$ in Rare Gas Lattices. The Journal of Chemical Physics 1975, 63, 794-804.

(60) Gerber, R. B.; Berkowitz, M. Role of Rotational and Translational Local Modes in Vibrational Relaxation in Solids: A Study of NH and ND in Solid Ar. Phys. Rev. Lett. 1977, 39, 1000-1004.

(61) Vanberkel, C.; Powell, M. J.; Franklin, A. R.; French, I. D. Quality Factor in A-Si-H Nip and Pin Diodes. J. Appl. Phys. 1993, 73, 5264-5268. 
(62) Street, R. A.; Krakaris, A.; Cowan, S. R. Recombination Through Different Types of Localized States in Organic Solar Cells. Adv. Funct. Mater. 2012, 22, 46084619.

(63) Street, R. A.; Song, K. W.; Northrup, J. E.; Cowan, S. Photoconductivity Measurements of the Electronic Structure of Organic Solar Cells. Phys. Rev. B 2011, 83, 165207.

(64) Street, R. A. Localized State Distribution and Its Effect on Recombination in Organic Solar Cells. Phys. Rev. B 2011, 84, 075208.

(65) Street, R. A.; Schoendorf, M.; Roy, A.; Lee, J. H. Interface State Recombination in Organic Solar Cells. Phys. Rev. B 2010, 81, 205307.

(66) Wetzelaer, G. J. A. H.; Kuik, M.; Blom, P. W. M. Identifying the Nature of Charge Recombination in Organic Solar Cells From Charge-Transfer State Electroluminescence. Adv. Energy Mater. 2012, 2, 1232-1237.

(67) Wetzelaer, G. A. H.; Kuik, M.; Lenes, M.; Blom, P. W. M. Origin of the DarkCurrent Ideality Factor in Polymer:Fullerene Bulk Heterojunction Solar Cells. Appl. Phys. Lett. 2011, 99, 153506.

(68) Kirchartz, T.; Deledalle, F.; Tuladhar, P. S.; Durrant, J. R.; Nelson, J. On the Differences Between Dark and Light Ideality Factor in Polymer:Fullerene Solar Cells. J. Phys. Chem. Lett. 2013, 4, 2371-2376.

(69) Walsh, A.; Scanlon, D. O.; Chen, S.; Gong, X. G.; Wei, S. H. Self-Regulation Mechanism for Charged Point Defects in Hybrid Halide Perovskites. Angew. Chem. Int. Ed. 2015, 54, 1791-1794.

(70) Nie, W.; Blancon, J. C.; Neukirch, A. J.; Appavoo, K.; Tsai, H.; Chhowalla, M.; Alam, M. A.; Sfeir, M. Y.; Katan, C.; Even, J. et al. Light-Activated Photocurrent Degradation and Self-Healing in Perovskite Solar Cells. Nat. Commun. 2016, 7, 11574.

(71) Long, R.; Prezhdo, O. V. Dopants Control Electron-Hole Recombination at Perovskite- $\mathrm{TiO}_{2}$ Interfaces: Ab Initio Time-Domain Study. Acs Nano 2015, 9, 11143-11155.

(72) Ferry, D. K. Semiconductors: Bonds and bands; IOP Publishing: Bristol, UK, 2013.

(73) Long, R.; Fang, W. H.; Prezhdo, O. V. Strong Interaction at the Perovskite/TiO 2 Interface Facilitates Ultrafast Photoinduced Charge Separation: A Nonadiabatic Molecular Dynamics Study. The Journal of Physical Chemistry C 2017, 121, 3797-3806.

(74) Alkauskas, A.; Buckley, B. B.; Awschalom, D. D.; Van de Walle, C. G. FirstPrinciples Theory of the Luminescence Lineshape for the Triplet Transition in Diamond NV Centres. New Journal of Physics 2014, 16, 073026. 
(75) Yang, J. H.; Shi, L.; Wang, L. W.; Wei, S. H. Non-Radiative Carrier Recombination Enhanced by Two-Level Process: A First-Principles Study. Sci. Rep. 2016, 6, 21712.

(76) Price, M. B.; Butkus, J.; Jellicoe, T. C.; Sadhanala, A.; Briane, A.; Halpert, J. E.; Broch, K.; Hodgkiss, J. M.; Friend, R. H.; Deschler, F. Hot-Carrier Cooling and Photoinduced Refractive Index Changes in Organic-Inorganic Lead Halide Perovskites. Nat. Commun. 2015, 6, 8420.

(77) Wu, X.; Tan, L. Z.; Shen, X.; Hu, T.; Miyata, K.; Trinh, M. T.; Li, R.; Coffee, R.; Liu, S.; Egger, D. A. et al. Light-Induced Picosecond Rotational Disordering of the Inorganic Sublattice in Hybrid Perovskites. Sci Adv 2017, 3.

(78) Yelon, A.; Movaghar, B.; Crandall, R. S. Multi-Excitation Entropy: Its Role in Thermodynamics and Kinetics. Rep. Prog. Phys. 2006, 69, 1145.

(79) Bozyigit, D.; Yazdani, N.; Yarema, M.; Yarema, O.; Lin, W. M. M.; Volk, S.; Vuttivorakulchai, K.; Luisier, M.; Juranyi, F.; Wood, V. Soft Surfaces of Nanomaterials Enable Strong Phonon Interactions. Nature 2016, 531, 618622.

(80) Niesner, D.; Wilhelm, M.; Levchuk, I.; Osvet, A.; Shrestha, S.; Batentschuk, M.; Brabec, C.; Fauster, T. Giant Rashba Splitting in $\mathrm{CH}_{3} \mathrm{NH}_{3} \mathrm{PbBr}_{3}$ OrganicInorganic Perovskite. Phys. Rev. Lett. 2016, 117, 126401.

(81) Motta, C.; El-Mellouhi, F.; Kais, S.; Tabet, N.; Alharbi, F.; Sanvito, S. Revealing the Role of Organic Cations in Hybrid Halide Perovskite $\mathrm{CH}_{3} \mathrm{NH}_{3} \mathrm{PbI}_{3}$. Nat. Commun. 2015, 6, 7026.

(82) Zhang, Z.; Long, R.; Tokina, M. V.; Prezhdo, O. V. Interplay Between Localized and Free Charge Carriers Can Explain Hot Fluorescence in the CH3NH3PbBr3 Perovskite: Time-Domain Ab Initio Analysis. J. Am. Chem. Soc. 2017, 139, 17327-17333.

(83) Dastidar, S.; Li, S.; Smolin, S. Y.; Baxter, J. B.; Fafarman, A. T. Slow ElectronHole Recombination in Lead Iodide Perovskites Does Not Require a Molecular Dipole. ACS Energy Lett. 2017, 2, 2239-2244.
Poglitsch
A.; Weber,
D.
Dynamic
Disorder in Methylammoniumtrihalogenoplumbates (II) Observed by Millimeter-Wave Spectroscopy. The Journal of Chemical Physics 1987, 87, 6373-6378.

(85) Markvart, T. Multiphonon recombination. In Recombination in Semiconductors, Landsberg, P. T., Ed.; Cambridge University Press: Cambridge, 2003; p 475. 